\title{
Overcoming Obstacles Facing the Contractors Sector in Saudi Arabia Using a Rank Correlation Method
}

\author{
Mansour N. Jadid* \\ Department of Building and Science Technology, University of Dammam, Saudi Arabia
}

\begin{abstract}
The contractors sector is considered to be one of the most important sectors in terms of its effects on economic development, stabilization, and urbanization. The main objective of this study was to explore and analyze the obstacles facing the contractors sector in Saudi Arabia from the contractors' point of view using Spearman's rank correlation method, where the dimensionality of ordinal data was reduced by factor analysis. This required a comprehensive investigation of the obstacles that prevented contractors from completing their jobs on time, or that led to them completing a lower quality job and losing money. This study only included Saudi contractors involved with building activities and it was based on data collection using comprehensive questionnaires that defined five main factors: managerial, financial, expansion, environmental and technical. Contractors who were qualified and registered by the Ministry of Municipal and Rural Affairs (MOMRA) were considered in this study. The study concentrated on the building sector, specifically the fields and activities defined by MOMRA. Identifying the obstacles that affect professional and technical activities should help to improve performance and address various problems. A digital interactive structural questionnaire was designed and distributed via the internet to collect responses from the participants where a Likert scale-based method was used. The results obtained using the questionnaires are presented as tables and figures.
\end{abstract}

Keywords: Contractor, correlation, factors, questionnaire, Saudi Arabia, spearman, likert.

\section{INTRODUCTION}

The construction industry is considered to be complicated and highly differentiated, and many factors affect the performance in this area. At present, construction projects in Saudi Arabia have become large and highly complex, so their management must be effective to ensure the success of these projects.

Saudi Arabia has witnessed an unprecedented boom in construction projects, including educational institutions, roads, bridges, reservoirs, water supply projects, the installation of communication equipment and networks, power stations, hospitals, and housing. The construction industry in Saudi Arabia includes many companies that employ nearly half a million people, including engineers, technicians, and workers. The market for the construction sector is very large but also highly risky, so it may require some re-organization. The present study was conducted to identify some of the obstacles faced by contractors. After Saudi Arabia joined the World Trade Organization (WTO), contractors had to reorganize their way of handling business with their clients. To keep pace with changes and developments in the domestic arena, as well as those in other countries, local contractors are facing competition from experienced international companies.

The construction sector is the major base of any world economy because it contributes greatly to the gross domestic product (GDP). The construction industry in Saudi Arabia is

*Address correspondence to this author at the Department of Building and Science Technology, University of Dammam, Saudi Arabia;

Tel: 00966505873655; Email: mnjadid@yahoo.com considered to be the second largest economic sector after the oil industry but the country's planned development depends on the revenues generated from its sale of oil. This sector is highly sensitive to economic cycles and the political environment, so there has been a significantly high rate of business failures in Saudi Arabia. This field is very large and is considered to be highly risky due to the failures related to its sensitivity to economic cycles and the high levels of competition. The author of the present study worked closely with contractors for many years to obtain an in-depth knowledge of the business of contractors and the obstacles they face on a daily basis.

\section{LITERATURE REVIEW}

A study [1] in Saudi Arabia for the causes of contractor failures concluded that there are four major causes of failure. These studies were based on questionnaires distributed to 67 Saudi contractors where statistical sampling was used to obtain the results. Another study [2] focused on the criteria used to evaluate the qualifications of contractor based on quantitative and qualitative methods when comparing bids from contractors. It was suggested the possibility to select a qualified, competent, and reliable contractor by evaluating his qualifications, economic and financial condition, technical capability, and skills in achieving relevant results. A model [3] was developed for benchmarking organizations based on the actual study of contractors in Saudi Arabia. An alternative empirical method [4] was proposed based on principal components analysis for prequalifying the contractors, which reduced the existing amount of multicollinearity and the dimensionality of the prequalification process. A different approach was proposed [5] who presented a model- 
ing methodology for prequalifying contractors, which demonstrated that a prequalification, multi-criteria decisionmaking model (PQDM) based on the analytical hierarchy process (AHP) could overcome the difficulties of the prequalification process. The problems that face small-and medium-sized contractors caused mainly by a lack of access to finance and late payments were explored by government sector [6]. Many obstacles were identified [7] in the contracting process in the West Bank of Palestine from the perspectives of clients, consultants, contractors and donor country institutions, which was related mainly to career ethics. Another perspective from the West Bank in Palestine was identified [8] who categorized the causes of contactor failure into three groups (financial, managerial, and external). A total of 84 contractors participated and 44 factors were identified in this study, which showed that fluctuations in construction material costs were the major obstacle, followed by delays in collecting small amounts of money from clients, a lack of experience in contracts, and other factors.

The present study concentrated on the building sector and the main objectives of the study were as follows:

- Analyzing the causes of problems faced by most contractors in Saudi Arabia.

- Identifying and analyzing the most important factors that create obstacles to contractors using Spearman's [9] rank correlation method.

- Discussing the results obtained and suggesting recommendations.

\section{METHODOLOGY}

Data were collected using a bilingual digital survey interactive questionnaire to identify the main obstacles faced in the contractors sector. The questionnaire was designed based on information collected during several years of dealing with contractors, including correspondence, meetings, discussions, documentation, contracts, literature, past experiences, and interviews. The obstacles that faced contractors in Saudi Arabia were investigated [1] who identified four main causes; environmental, managerial, financial, and expansion. The present study concentrated on Saudi contractors engaged in building activities and was based on data collection using questionnaires developed from the concepts proposed [1], which were modified to consider the changing conditions of current technology and an increased number of questionnaires were used, as shown in Table 1. To keep up with technological advances a fifth item was introduced under the heading technical causes.

This method was quantitative because it focused on measuring the significance of causes related to the obstacles faced by contractors. The Ministry of Municipal and Rural Affairs [10] classified Saudi contractors into 27 construction and maintenance fields, where each field was graded one to five based on the contractors' financial capabilities.

A digital interactive structural questionnaire was designed and distributed to contractors via the internet so they could express their views regarding the obstacles they were facing. A total of 71 contractors responded to the questionnaires and the 74 questions were divided into five main causes, as follows:

- Environmental (11)

- Financial (9)

- Expansion (7)

- Technical (8)

- Managerial (39)

In addition, general data were collected regarding the company information: specialty, nationality, years of experience, annual company income, average number of workers, company grade, number of projects conducted, and bids awarded.

A Likert sampling method [11] was used for data collection. The information collection and analysis process comprised the following:

- General overview of the contractors.

- Distribution and collection of the completed forms via an interactive interface.

- Limited site visits to verify the information gathered based on interviews and discussions with contractors, without any interference from the clients or consultants.

- Data analysis and identification of the most relevant factors that created obstacles to contractor.

- Analysis of the results to compare the opinions of contractors using a scientific method.

\section{EXPLORATION AND ANALYSIS}

This study analyzed obstacles faced by contractors Saudi Arabia, i.e., contractors classified [10] as grade one who

Table 1. Comparison Between the Number of Causes Proposed [1] and Those Analyzed in the Present Study

\begin{tabular}{|c|c|c|}
\hline Causes & Number of causes proposed by [1] & Number of causes proposed in the present study \\
\hline \hline Environmental & 4 & 11 \\
\hline Financial & 7 & 9 \\
\hline Expansion & 7 & 7 \\
\hline Managerial & 16 & 39 \\
\hline Technical & $-3----$ & 8 \\
\hline Total & $\mathbf{3 4}$ & $\mathbf{7 4}$ \\
\hline
\end{tabular}


were eligible to conduct projects valued at more than 75 million US dollars (280 million Saudi riyals).

The Likert scale described [11] was used in the questionnaire to measure the opinions of contractors. The Likert scale used [11] is a suitable method for analyzing the strength of an opinion because it uses numbers to represent implicit meanings. The data were all based on the responses made by the contractors. The factors were presented to the contractors and scored using a five-point scale, which ranged from 1 (cannot decide) to 5 (very strongly influence). Each attribute was evaluated as follows:

- Cannot decide (1)

- Not influenced (2)
- Slightly influenced (3)

- Influenced (4)

- Very strongly influenced (5)

Spearman's [9] rank correlation method was used to test whether the relationship between the strength and direction of two variables was monotonically related. The method used to calculate the correlations is shown in (Fig. 1). The variance/covariance matrix for two variables $X$ and $Y$ was simplified by Spearman's [9] as follows:

$$
\sigma_{x y}=\frac{1}{n} \sum_{i=1}^{n}\left(x_{i}-\bar{x}\right)\left(y_{i}-\bar{y}\right)
$$

The $k \times k$ variance / covariance matrix is calculated using the following formula:

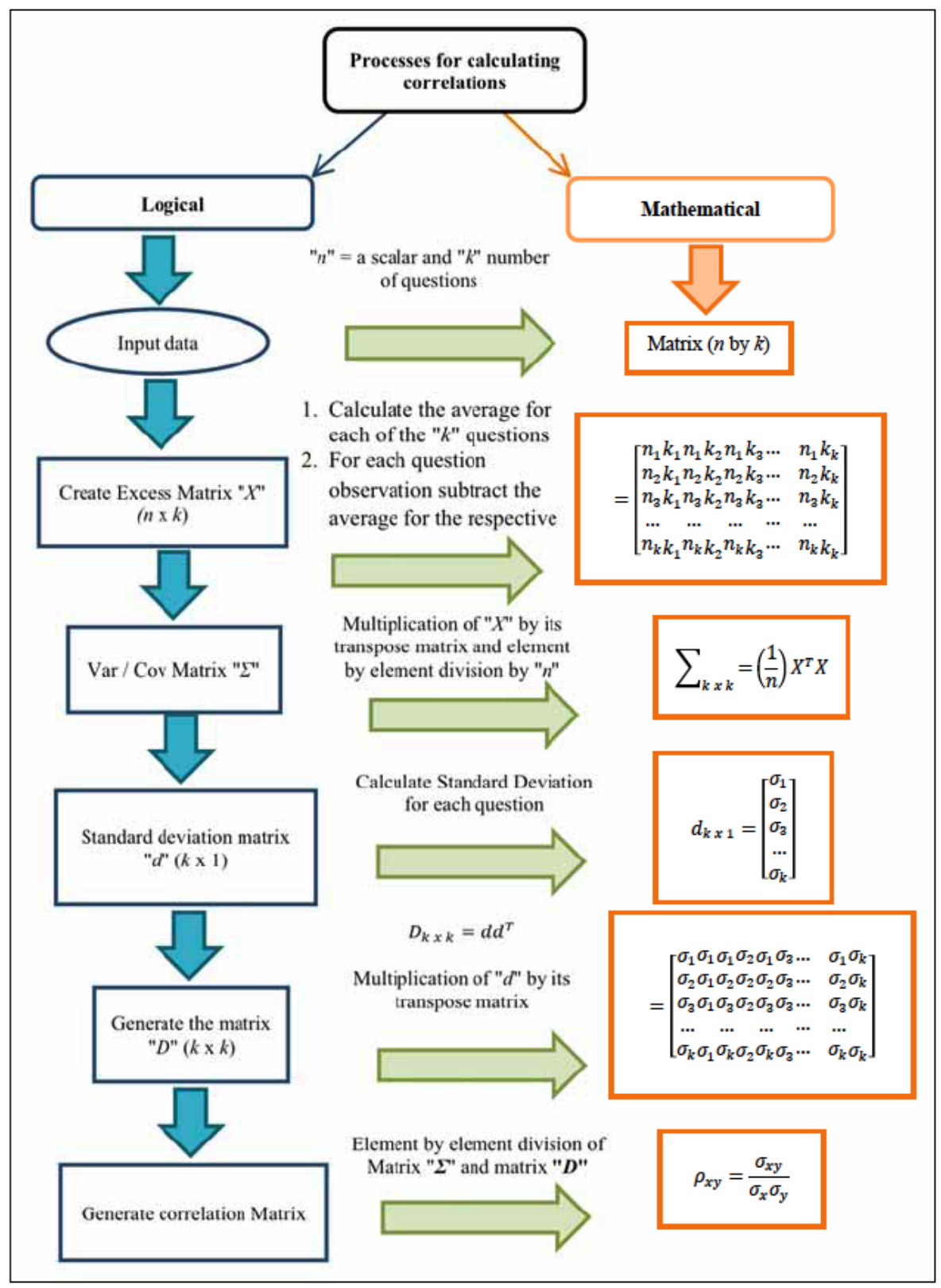

Fig. (1). Logical process and mathematical correlation calculations. 
$\sum_{k x k}=\left(\frac{1}{n}\right) X^{T} X$

where

$\sigma_{x y}=$ variance / covariance matrix

$n=$ a scalar

$X=(n \times k)$ excess matrix

$X^{T} X=$ the multiplication of matrix $X$ by its transpose.

$k=$ number of questions

The steps used to evaluate the above equation were as follows (e.g., for the technical causes questions).

1. Create an $(n \times k)$ matrix, where " $n$ " is the number of contractors and " $k$ " is the number of questions.

2. Calculate the average for each of the " $k$ " questions.

3. For each question observation, subtract the average for the respective questions.

4. Define a new $(n \times k)$ matrix as the excess matrix " $X$ ".

The scatter plot matrix captured all of the aforementioned statistical methods and the analysis was carried out using statistical analysis [12] while the data manipulation was performed using Microsoft excel.

\section{RESULTS AND DISCUSSION}

Difficulties have been encountered in carrying out this work, mainly information requested from the contractors. Contractors were hesitated at the beginning to provide vital information about their companies. Few of them found the automated process of filling the online process difficult and they could not complete the questionnaire. Another issue was observed due to the emails returned to the incorrect email addresses. Moreover, bulk emails were transferred to the spam or junk folders in which emails were never opened.

In this work, a total of 74 causes were analyzed with the managerial cause has maximum percentage of 52.703 of the total causes, followed by percentage of $14.865,12.162$, 10.811 and 9.495 for environmental, financial, expansion and technical causes respectively.

The factors that affect the obstacles facing the contractors sector were analyzed from the perspectives of the contractors using Spearman's [9] rank correlation method. The results for the different sets of questions are presented as tables and figures, which are explained as follows.

\subsection{Environmental Factors}

For the environmental questions, Table 1a shows that the modal frequencies mainly scored 4 (influenced). For q5 (country at war) and q10 (delay in collecting money from clients), the modal response was 5 (very strongly influenced).

The environmental questions in Table $\mathbf{1 b}$ show that there were correlations $(>0.5)$ between $\mathrm{q} 3$ and $\mathrm{q} 6, \mathrm{q} 3$ and $\mathrm{q} 8, \mathrm{q} 6$ and $\mathrm{q} 8$, and $\mathrm{q} 8$ and $\mathrm{q} 11$. In the scatter plot matrix shown in (Fig. 2), these are represented by an elongated and relatively narrow ellipse. Questions with few or no correlations are represented by circles in the scatter plot matrix.

\subsection{Financial Factors}

For the financial questions, Table 2a shows that the modal frequencies mainly scored 4 (influenced). For q4 (poor estimation practice) and q9 (delay in receiving money from the Ministry of Finance), the modal response was 5 (very strongly influenced).

The financial questions in Table $\mathbf{2 b}$ show that there were correlations $(>0.5)$ between $\mathrm{q} 3$ and $\mathrm{q} 6, \mathrm{q} 6$ and $\mathrm{q} 7$, and $\mathrm{q} 7$ and q8. These correlations are also reflected in the scatter plot shown in (Fig. 3).

\subsection{Expansion Factors}

For the expansion questions, Table 3a shows that the modal frequencies mainly scored 4 (influenced). For q2

Table 1a. Modal Frequencies for the Environmental Questions

\begin{tabular}{|c|c|c|c|c|c|}
\hline Question ID & Environmental Questions & $\begin{array}{c}\text { Modal } \\
\text { response }\end{array}$ & Number & Total & Percentage \\
\hline $\mathrm{q} 1$ & National slump in the economy & 4 & 27 & 71 & 38.0 \\
\hline q3 & Owner involvement in construction phase & 4 & 30 & 71 & 42.3 \\
\hline $\mathrm{q} 4$ & Bad weather & 3 & 36 & 71 & 50.7 \\
\hline q6 & Fluctuation of Materials prices & 4 & 37 & 71 & 52.1 \\
\hline q7 & Easing the recruitment restrictions & 4 & 32 & 71 & 45.1 \\
\hline q8 & Increase the prices of equipment & 4 & 45 & 71 & 63.4 \\
\hline q9 & Political decisions & 3,4 & 20 & 71 & 28.2 \\
\hline
\end{tabular}


Table 1b. Spearman's [9] Correlation Matrix for the Environmental Questions

\begin{tabular}{|c|c|c|c|c|c|c|c|c|c|c|c|}
\hline VAR1 & 1 & & & & & & & & & & \\
\hline VAR3 & 0.321 & 0.472 & 1 & & & & & & & & \\
\hline VAR6 & 0.352 & 0.423 & 0.531 & 0.261 & 0.133 & 1 & & & & & \\
\hline VAR7 & 0.11 & 0.401 & 0.344 & 0.005 & 0.106 & 0.396 & 1 & & & & \\
\hline VAR8 & 0.484 & 0.388 & 0.537 & 0.334 & 0.284 & 0.502 & 0.319 & 1 & & & \\
\hline
\end{tabular}

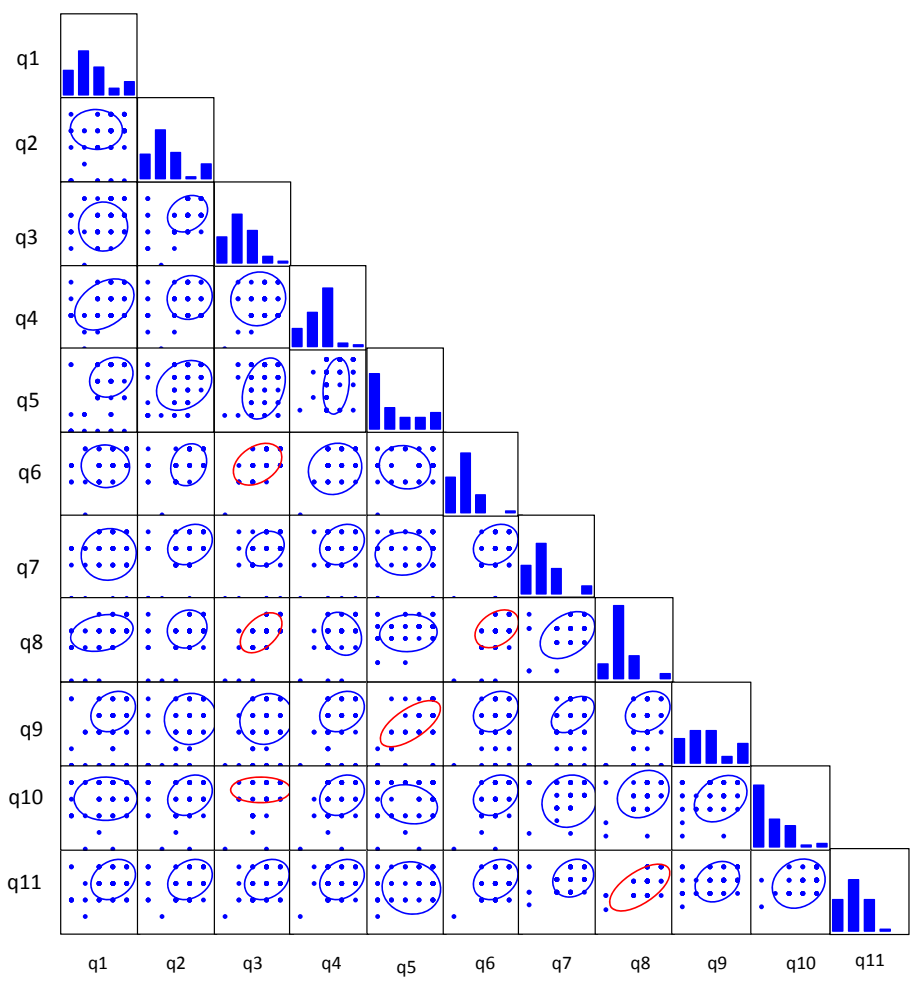

Fig. (2). Scatter plot matrix for the environmental questions.

(opening a regional office), the modal response was 3 (slightly influenced).

The expansion questions in Table $\mathbf{3 b}$ show that there were correlations $(>0.5)$ between $\mathrm{q} 1$ and $\mathrm{q} 2$, $\mathrm{q} 1$ and $\mathrm{q} 2, \mathrm{q} 1$ and $\mathrm{q} 4, \mathrm{q} 3$ and $\mathrm{q} 4$. These correlations are also reflected in the scatter plot shown in (Fig. 4).

\subsection{Technical Factors}

For the technical questions, Table $4 \mathbf{a}$ shows that the modal frequencies mainly scored 4 (influenced). For q5 (techni- cal and managerial rapid actions from the site supervision consultant team), the modal response was 5 (very strongly influenced). None of pairs of the technical questions had correlations $>0.5$, which is shown in Table $\mathbf{4 b}$. The scatter plot for technical factor is shown in (Fig. 5).

\subsection{Managerial Factors}

For the managerial questions, Table 5a shows that the modal frequencies mainly scored 4 (influenced). Some questions had model frequencies of 3 or 5 . 
Table2a. Modal Frequencies for the Financial Questions

\begin{tabular}{|c|c|c|c|c|c|}
\hline Question ID & Financial Questions & Modal response & Number & Total & Percentage \\
\hline q1 & Low margin profit due to competition & 4 & 36 & 71 & 50.7 \\
\hline q3 & Cash flow management & 4 & 30 & 71 & 42.3 \\
\hline $\mathrm{q} 4$ & Poor estimation practices & 5 & 46 & 71 & 64.8 \\
\hline q6 & Controlling equipment cost and usage & 4 & 35 & 71 & 49.3 \\
\hline q7 & Evaluate project profit in one fiscal year & 3,4 & 27 & 71 & 38.0 \\
\hline q8 & Employee benefits and compensations & 4 & 32 & 71 & 45.1 \\
\hline q9 & Delay in receiving money from the Ministry of finance & 5 & 39 & 70 & 55.7 \\
\hline
\end{tabular}

Table 2b. Spearman's [9] Correlation Matrix for the Financial Questions

\begin{tabular}{|c|c|c|c|c|c|c|c|c|c|}
\hline VAR1 & 1 & & & & & & & & \\
\hline VAR3 & 0.252 & 0.213 & 1 & & & & & & \\
\hline VAR4 & 0.264 & 0.081 & 0.392 & 1 & & & & & \\
\hline VAR6 & 0.153 & 0.028 & 0.516 & 0.262 & 0.168 & 1 & & & \\
\hline VAR7 & 0.25 & 0.183 & 0.435 & 0.161 & 0.359 & 0.58 & 1 & & \\
\hline VAR8 & 0.264 & 0.067 & 0.188 & 0.18 & 0.31 & 0.261 & 0.584 & 1 & \\
\hline VAR9 & 0.169 & 0.132 & 0.369 & 0.215 & 0.375 & 0.153 & 0.257 & 0.26 & 1 \\
\hline
\end{tabular}

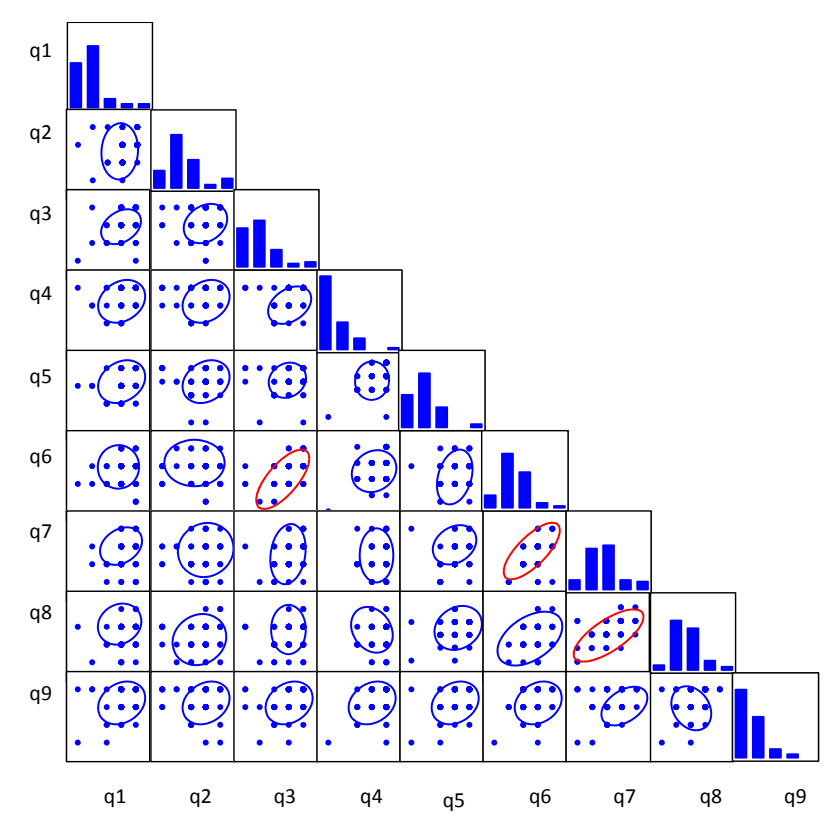

Fig. (3). Scatter plot matrix for the financial questions. 
Table 3a. Modal Frequencies for the Expansion Questions

\begin{tabular}{|c|c|c|c|c|}
\hline Question ID & Expansion Questions & $\begin{array}{c}\text { Modal } \\
\text { response }\end{array}$ & Number & Total \\
\hline \hline q1 & Expansion into new graphical locations & 4 & 37 & 71 \\
\hline q2 & Opening a regional office & 3 & 31 & 71 \\
\hline q3 & Increased number of projects & 4 & 33 & 71 \\
\hline q4 & Increased size of projects & 4 & 34 & 71 \\
\hline q5 & Change in the type of work & 3,4 & 28 & 71 \\
\hline q6 & Lack of managerial development or maturity as the com- & 4 & 31 & 71 \\
\hline q7 & Change from private to public or vice versa & 4 & 28 & 73.7 \\
\hline
\end{tabular}

Table 3b. Spearman's [9] Correlation Matrix for the Expansion Questions

\begin{tabular}{|c|c|c|c|c|c|c|}
\hline & VAR1 & VAR2 & VAR3 & VAR4 & VAR5 & VAR6 \\
\hline \hline VAR1 & 1 & & & & & \\
\hline VAR2 & $\mathbf{0 . 5 3 5}$ & 1 & & & & \\
\hline VAR3 & $\mathbf{0 . 5 3 9}$ & $\mathbf{0 . 5 7 2}$ & 1 & & & \\
\hline VAR4 & $\mathbf{0 . 5 1 1}$ & 0.454 & $\mathbf{0 . 8 1}$ & 1 & & \\
\hline VAR5 & 0.439 & 0.346 & 0.379 & 0.443 & 1 & \\
\hline VAR6 & 0.198 & 0.203 & 0.143 & 0.085 & 0.351 & 1 \\
\hline VAR7 & 0.366 & 0.357 & 0.105 & 0.216 & 0.324 & 0.299 \\
\hline
\end{tabular}

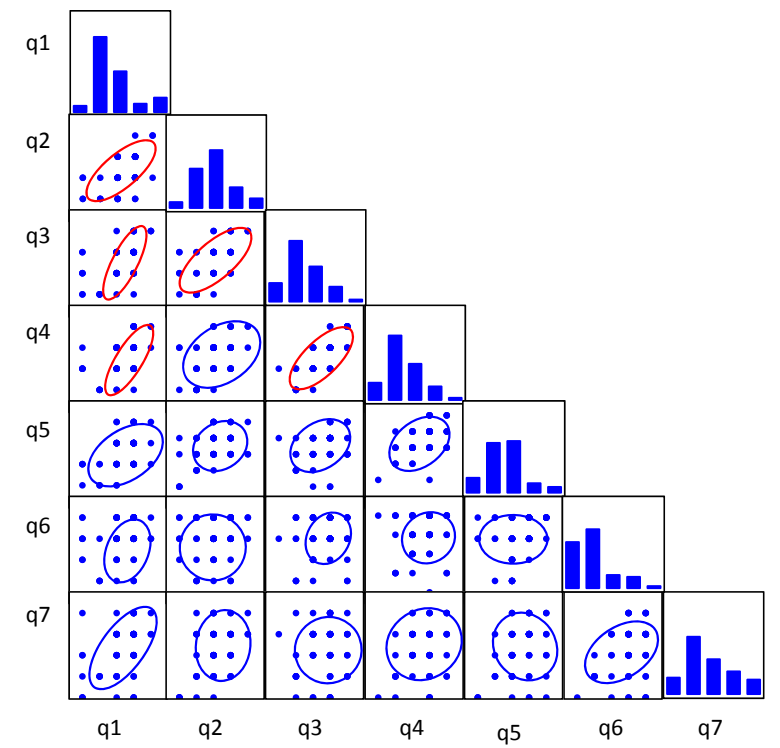

Fig. (4). Scatter plot matrix for the expansion questions. 
Table 4a. Modal Frequencies for the Technical Questions

\begin{tabular}{|c|c|c|c|c|c|}
\hline Question ID & Technical Questions & $\begin{array}{c}\text { Modal } \\
\text { response }\end{array}$ & Number & Total & Percentage \\
\hline q1 & Owner end user requirements during construction & 4 & 34 & 70 & 48.6 \\
\hline $\mathrm{q} 2$ & $\begin{array}{l}\text { Lack or discrepancy of information in drawings, specifications } \\
\text { and all tender documents }\end{array}$ & 4 & 33 & 70 & 47.1 \\
\hline q5 & $\begin{array}{l}\text { Technical and managerial quick actions from site supervision consultant } \\
\text { team }\end{array}$ & 5 & 32 & 70 & 45.7 \\
\hline q6 & Quick response from ( contractor) team & 4 & 39 & 70 & 55.7 \\
\hline q7 & Building technology machines and tools & 4 & 33 & 70 & 47.1 \\
\hline
\end{tabular}

Table 4b. Spearman's [9] Correlation Matrix for the Technical Questions

\begin{tabular}{|c|c|c|c|c|c|c|c|c|}
\hline & VAR1 & VAR2 & VAR3 & VAR4 & VAR5 & VAR6 & VAR7 & VAR8 \\
\hline VAR1 & 1 & & & & & & & \\
\hline VAR2 & 0.457 & 1 & & & & & & \\
\hline VAR3 & 0.419 & 0.293 & 1 & & & & & \\
\hline VAR4 & 0.272 & 0.268 & 0.191 & 1 & & & & \\
\hline VAR5 & 0.252 & 0.213 & 0.393 & 0.358 & 1 & & & \\
\hline VAR6 & 0.195 & 0.07 & 0.267 & 0.267 & 0.423 & 1 & & \\
\hline VAR7 & 0.148 & 0.208 & 0.172 & 0.423 & 0.348 & 0.488 & 1 & \\
\hline VAR8 & 0.096 & 0.291 & 0.377 & 0.463 & 0.282 & 0.249 & 0.409 & 1 \\
\hline
\end{tabular}

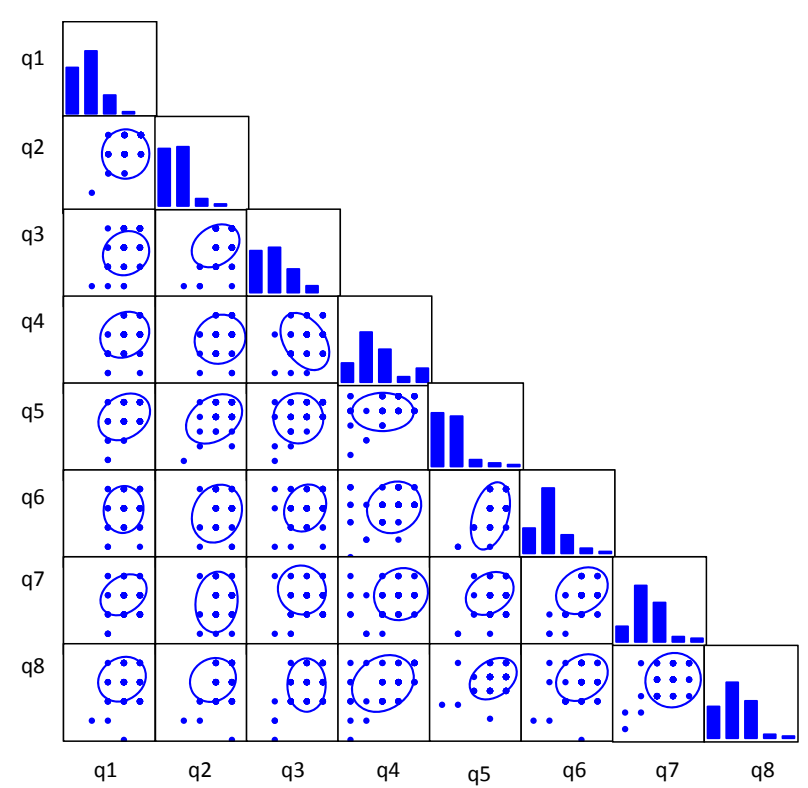

Fig. (5). Scatter plot matrix for the technical questions. 
Table 5a. Modal Frequencies for the Managerial Questions

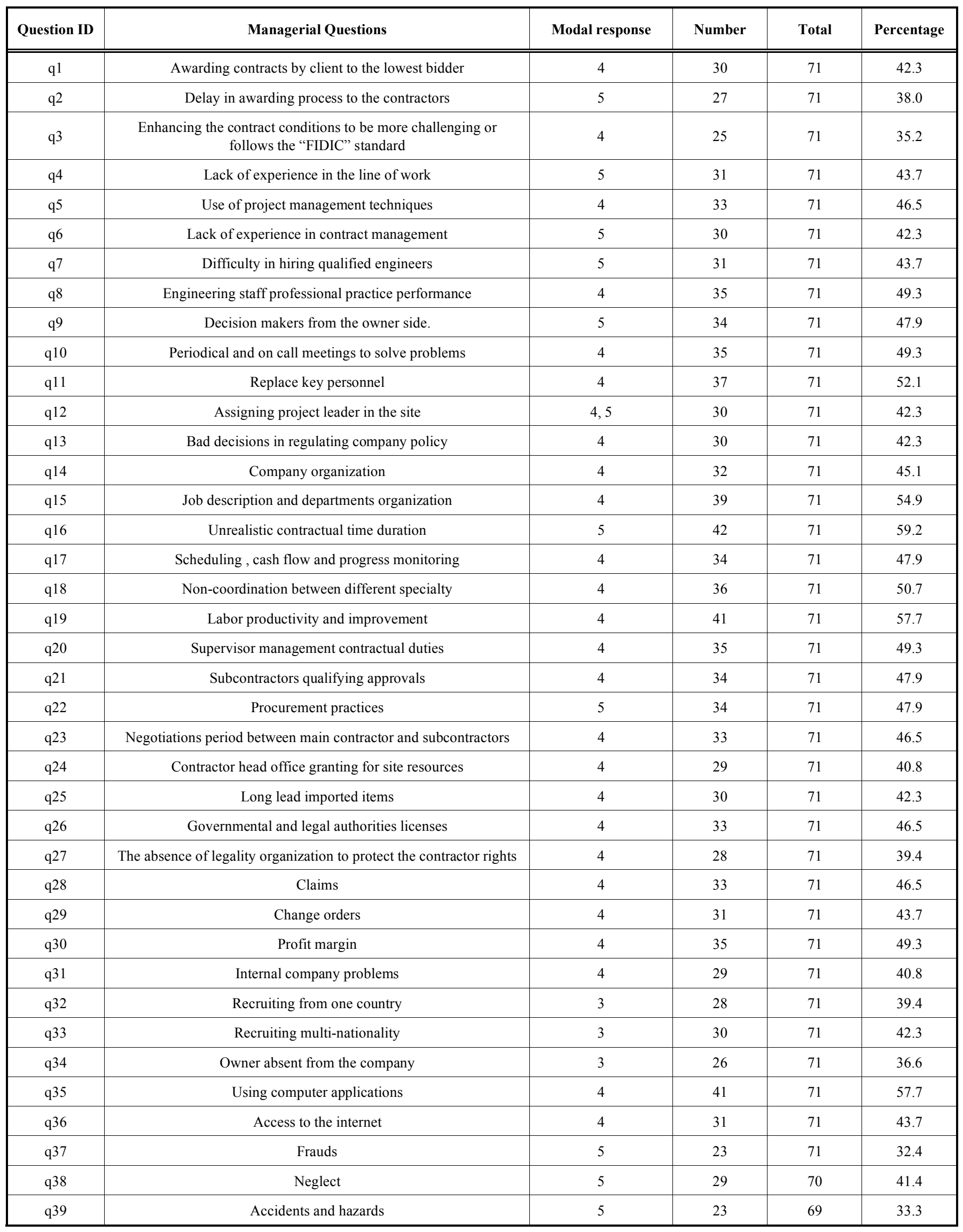




\begin{tabular}{|c|c|c|c|c|c|c|c|c|c|c|c|c|c|c|c|c|c|c|c|c|c|c|c|c|c|c|c|c|c|c|c|c|c|c|c|c|c|c|c|}
\hline \begin{tabular}{|l|}
\multirow{3}{*}{} \\
\end{tabular} & \begin{tabular}{|l|} 
\\
\end{tabular} & \begin{tabular}{|l|}
\multirow{G}{G}{} \\
\end{tabular} & 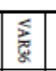 & 志 & 产 & \begin{tabular}{|l|}
\multirow{z}{*}{} \\
\end{tabular} & 畓 & $\widehat{\underline{\underline{E}}}$ & 㢇 & 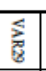 & 爵 & & 密 & 銮 & 営 & 飒 & 离 & 憲 & 茞 & 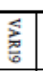 & 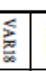 & 兽 & 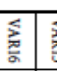 & & & $\sum_{\bar{\omega}}^{\vdots}$ & 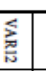 & $\underline{\underline{\underline{x}}}$ & 总 & 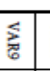 & $\aleph_{\infty}^{\lessgtr}$ & 庼 & 婄 & 感 & 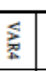 & 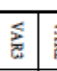 & 旋 & & \\
\hline$\frac{1}{\vdots}$ & 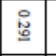 & $\frac{0}{9}$ & 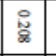 & \begin{tabular}{|l|} 
\\
$x$
\end{tabular} & $\frac{\mathrm{O}}{2}$ & \begin{tabular}{|l|}
$\frac{1}{8}$ \\
\end{tabular} & 蛅 & $\dot{0}$ & 㟧 & 兽 & $\frac{\circ}{3}$ & $:$ & 8 & 岁 & $\frac{O}{b}$ & 离 & $\stackrel{8}{8}$ & 잉 & $\stackrel{i}{\square}$ & $:$ & $\frac{1}{3}$ & $\frac{8}{8}$ & 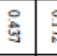 & 音 & $\stackrel{\square}{\circ}$ & $\frac{\circ}{8}$ & 章 & 妾 & $\stackrel{\circ}{\mathrm{B}}$ & $\stackrel{\square}{\sharp}$ & $\mathbb{\&}$ & 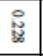 & 要 & 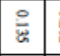 & 畩 & 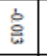 & $\underline{\Xi}$ & & 畜 \\
\hline$\frac{\circ}{4}$ & \begin{tabular}{|l|}
\multirow{3}{*}{} \\
\end{tabular} & 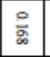 & 言 & $\begin{array}{ll}8 \\
8\end{array}$ & 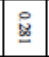 & . & 离 & $\stackrel{\circ}{\square}$ & 今 & $\stackrel{\circ}{2}$ & 总 & : & 嫚 & 总 & 要 & $\stackrel{3}{3}$ & 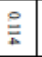 & 景 & 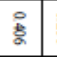 & 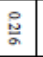 & 产 & 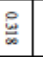 & 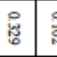 & 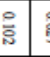 & . & $\stackrel{\xi}{\xi}$ & : & 鹿 & 是 & 总 & $\stackrel{8}{8}$ & 奠 & : & 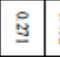 & 恙 & $\stackrel{8}{\underline{\theta}}$ & - & & 忢 \\
\hline$\frac{b}{\underline{z}}$ & $\frac{\circ}{9}$ & 号 & 离 & 疃 & $\stackrel{9}{\sharp}$ & 居 & $\frac{9}{\bar{x}}$ & $\frac{\circ}{\circ}$ & $\frac{9}{2}$ & 童 & 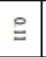 & 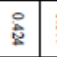 & : & 잉 & 峦 & 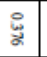 & $\frac{1}{8}$ & $\frac{\mathrm{q}}{\mathrm{i}}$ & $\frac{9}{9}$ & 澱 & $\frac{8}{\dot{a}}$ & $\dot{8}$ & $\begin{array}{lll} \\
\end{array}$ & $\frac{1}{8}$ & $\mathscr{B}$ & 多 & $\%$ & 8 & $\stackrel{g}{G}$ & 惫 & $\stackrel{9}{3}$ & $\stackrel{B}{*}$ & $\stackrel{4}{\sharp}$ & $\mid$\begin{tabular}{l|}
$\mathscr{8}$ \\
\end{tabular} & $\mathscr{8}$ & - & & & 产 \\
\hline$\ddot{g}$ & \begin{tabular}{|l|l|} 
\\
\end{tabular} & 要 & : & 譄 & 总 & $\frac{\circ}{\circ}$ & 㥕 & 虽 & 영 & $\frac{O}{w}$ & $:$ & 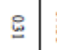 & : & 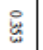 & 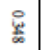 & 总 & $\frac{2}{0}$ & 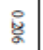 & $\frac{2}{0}$ & $\stackrel{9}{\$}$ & $\stackrel{8}{b}$ & 현 & \begin{tabular}{l|l}
5 \\
5
\end{tabular} & 粍 & 产 & 商 & : & 总 & $\stackrel{巳 g}{*}$ & 总 & $\stackrel{\square}{\circ}$ & 恋 & 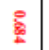 & 䇋 & - & & & & $\hat{t}$ \\
\hline 8 & 惫 & 兽 & 9 & 音 & 惫 & \begin{tabular}{|l|}
$\frac{1}{4}$ \\
\end{tabular} & $\stackrel{8}{8}$ & \&: & $\frac{\circ}{2}$ & 惫 & \% & $\frac{\circ}{4}$ & $\frac{9}{1}$ & 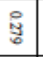 & 要 & $\stackrel{8}{\underline{8}}$ & $\stackrel{\leftrightarrow}{\mathscr{E}}$ & $\stackrel{\mathbb{\alpha}}{\underline{x}}$ & : & $\stackrel{\Xi}{\Xi}$ & 兽 & 厸 & $\begin{array}{l}8 \\
\end{array}$ & 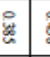 & \& & 총 & 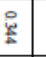 & 总 & $\frac{2}{2}$ & $\stackrel{8}{.0}$ & 愈 & 惫 & 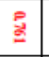 & - & & & & & 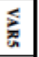 \\
\hline 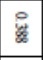 & \begin{tabular}{|l|}
$\circ$ \\
\end{tabular} & $\because$ & \& & 童 & $\begin{array}{l} \\
\dot{3}\end{array}$ & \begin{tabular}{|l|l|} 
\\
\end{tabular} & $\ddot{*}$ & 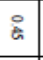 & $\frac{1}{3}$ & 总 & 咅 & 8 & 8 & 垔 & 8 & 曼 & 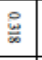 & 8 & $\dot{B}$ & $\stackrel{\circ}{\stackrel{\circ}{*}}$ & 善 & 畜 & \begin{tabular}{l|l}
$\circ$ \\
\multirow{2}{*}{8}
\end{tabular} & \& & 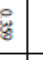 & 苑 & \& & 恶 & 葛 & $\stackrel{8}{\underline{z}}$ & 过 & 妾 & - & & & & & & 产 \\
\hline 范 & 居 & 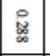 & \begin{tabular}{|l} 
\\
\end{tabular} & \begin{tabular}{|l|} 
\\
$\infty$
\end{tabular} & \begin{tabular}{|c|c|} 
\\
\end{tabular} & \begin{tabular}{|l|} 
\\
\end{tabular} & $\stackrel{\circ}{\circ}$ & 竞 & 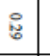 & $\stackrel{8}{0}$ & $\stackrel{\circ}{\circ}$ & : & 善 & $\dot{\dot{\phi}}$ & 昜 & 惫 & 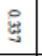 & 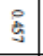 & $\stackrel{8}{0}$ & 怘 & 产 & 8 & : & 呟 & : & $\stackrel{\AA}{\xi}$ & 咅 & 星 & 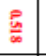 & 啳 & 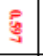 & - & & & & & & & $\stackrel{5}{3}$ \\
\hline 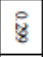 & 若 & : & 垔 & \begin{tabular}{|l|} 
\\
\end{tabular} & $\%$ & 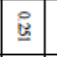 & $\underline{\underline{\theta}}$ & $\underline{\mathrm{B}}$ & 8 & $\ddot{s}$ & 总 & 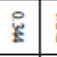 & 8 & 量 & 弯 & $\&$ & 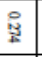 & $\$$ & 曷 & $\ddot{i}$ & 私 & 8 & \begin{tabular}{l|l}
5 \\
5
\end{tabular} & 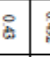 & 里 & 㫧 & 恶 & 영 & 展 & 离 & - & & & & & & & & $\frac{1}{8}$ \\
\hline 蛋 & 䬺 & $\frac{\mathscr{O}}{\infty}$ & 兽 & 奚 & 总 & $\stackrel{\circ}{\circ}$ & $\stackrel{8}{8}$ & 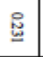 & 景 & $\mathrm{E}$ & $\stackrel{\mathscr{g}}{\mathrm{E}}$ & $\stackrel{8}{\circ}$ & $\stackrel{1}{ \pm}$ & 音 & 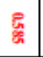 & 惫 & 紊 & 紊 & 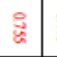 & 입 & 愛 & 变 & 蛅 & 咅 & 잉 & 忌 & 厎 & 是 & 至 & - & & & & & & & & & 爱 \\
\hline 紊 & $\begin{array}{l}\circ \\
\dot{8} \\
\end{array}$ & \begin{tabular}{|l|} 
\\
$\dot{3}$ \\
\end{tabular} & $\frac{9}{3}$ & 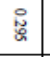 & $\ddot{G}$ & $\frac{\circ}{\bar{B}}$ & 离 & $\frac{0}{8}$ & 范 & 妾 & $\stackrel{9}{\%}$ & : & $\frac{\%}{2}$ & $\mathscr{\sharp}$ & 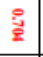 & 惫 & 总 & $\because$ & $\begin{array}{l}8 \\
\frac{1}{\omega}\end{array}$ & 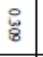 & 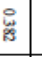 & $\stackrel{i}{\xi}$ & $\frac{1}{2}$ & $\begin{array}{ll} \\
\end{array}$ & $\begin{array}{l}\text { 音 } \\
\end{array}$ & 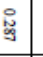 & 颜 & $\begin{array}{l}\text { 章 } \\
\end{array}$ & - & & & & & & & & & & 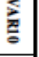 \\
\hline : & $\begin{array}{l} \\
\end{array}$ & \begin{tabular}{|l|} 
\\
\end{tabular} & $\stackrel{一}{\bar{E}}$ & : & \begin{tabular}{|l|l} 
\\
\\
\end{tabular} & \begin{tabular}{|l|} 
\\
\end{tabular} & 变 & 浐 & 高 & $\stackrel{8}{8}$ & $\begin{array}{ll} \\
\end{array}$ & 总 & 总 & $\dot{0}$ & 弯 & $\Xi$ & $\frac{8}{ \pm}$ & $\Xi$ & 总 & 童 & $\begin{array}{l}8 \\
\\
5\end{array}$ & 䌟 & 8 & 8 & : & : & $\underline{\underline{h}}$ & - & & & & & & & & & & & 庰 \\
\hline$\frac{0}{4}$ & $\begin{array}{l}0 \\
1\end{array}$ & 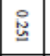 & 总 & 惫 & $\frac{1}{3}$ & \begin{tabular}{|l|} 
\\
\end{tabular} & 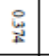 & $\stackrel{\circ}{\circ}$ & i & $\begin{array}{l}8 \\
\text { i }\end{array}$ & 8 & $\ddot{\ddot{g}}$ & $\frac{\mathscr{O}}{6}$ & $\stackrel{\mathscr{H}}{\sharp}$ & 童 & : & 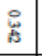 & : & $\begin{array}{l}8 \\
\$\end{array}$ & 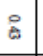 & 彩 & 总 & 通 & 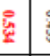 & 妾 & 站 & - & & & & & & & & & & & & 送 \\
\hline : & \begin{tabular}{|l} 
\\
$\Xi$
\end{tabular} & \& & 音 & : & $\stackrel{\circ}{.1}$ & \begin{tabular}{|l|} 
\\
\end{tabular} & 妾 & 离 & 은 & $\frac{\circ}{\circ}$ & $\stackrel{\circ}{\infty}$ & $\frac{\circ}{2}$ & $\frac{8}{3}$ & $\stackrel{8}{Z}$ & \& & $\stackrel{8}{\sharp}$ & 总 & $\frac{1}{8}$ & \& & $\stackrel{\circ}{\circ}$ & 童 & 量 & $\begin{array}{lll}8 \\
\end{array}$ & $\begin{array}{ll}0 \\
\dot{2}\end{array}$ & 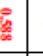 & - & & & & & & & & & & & & & 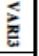 \\
\hline 8 & \begin{tabular}{|l|} 
\\
$\vdots$ \\
\end{tabular} & $\begin{array}{l} \\
\frac{g}{2} \\
\end{array}$ & \begin{tabular}{|l|} 
\\
\end{tabular} & 总 & $\begin{array}{l} \\
\end{array}$ & 导 & 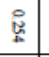 & $\stackrel{8}{\square}$ & $\stackrel{\circ}{\underline{-}}$ & 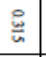 & $\stackrel{\varrho}{\vdots}$ & $\stackrel{0}{0}$ & 递 & $\underline{z}$ & 鯜 & $\because$ & 范 & 8 & 盖 & 8 & 嗛 & $:$ & 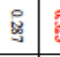 & 惫 & - & & & & & & & & & & & & & & 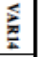 \\
\hline 产 & i & \begin{tabular}{|l|} 
\\
\end{tabular} & \begin{tabular}{|l} 
\\
:
\end{tabular} & 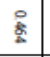 & \begin{tabular}{|l} 
\\
$\underline{\Phi}$ \\
\end{tabular} & 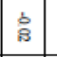 & 总 & 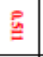 & 总 & 焉 & 总 & $\frac{\mathscr{g}}{2}$ & $\underline{\underline{\underline{X}}}$ & : & 景 & 妾 & 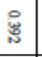 & $\stackrel{\circ}{0}$ & : & 密 & 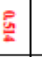 & 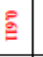 & $\begin{array}{lll}0 & -\end{array}$ & - & & & & & & & & & & & & & & & 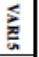 \\
\hline \& & 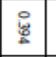 & \begin{tabular}{|l|} 
\\
\end{tabular} & \& & $\frac{\circ}{10}$ & \begin{tabular}{|l|} 
\\
\end{tabular} & \begin{tabular}{|l|l|}
\multirow{3}{*}{} \\
\end{tabular} & $\$$ & $\stackrel{\circ}{5}$ & 变 & $\frac{\mathrm{g}}{\frac{\mathrm{g}}{2}}$ & 总 & \% & 总 & 点 & 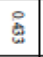 & $\stackrel{\leftrightarrow}{*}$ & 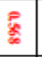 & 焉 & 8 & 站 & $\stackrel{.}{\dot{E}}$ & $\stackrel{0}{\circ}$ & - & & & & & & & & & & & & & & & & 序 \\
\hline \& & \begin{tabular}{|l|} 
离 \\
\end{tabular} & \begin{tabular}{|l|} 
\\
\end{tabular} & : & 恙 & 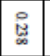 & \& & $\stackrel{8}{*}$ & $\begin{array}{l} \\
\vdots\end{array}$ & $\underline{\underline{\theta}}$ & 思 & 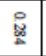 & $\frac{8}{2}$ & $\frac{E}{5}$ & 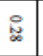 & 悹 & $\stackrel{9}{\dddot{m}}$ & 영 & 弯 & \& & : & 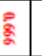 & - & & & & & & & & & & & & & & & & & 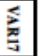 \\
\hline 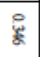 & 要 & $\begin{array}{ll} \\
\end{array}$ & 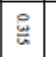 & : & 8 & \begin{tabular}{|l|l|}
\multirow{2}{*}{} \\
\end{tabular} & (3) & $\stackrel{\circ}{\circ}$ & 产 & 㝇 & 总 & 8 & 8 & i & 8 & 紊 & $\therefore$ & 商 & 8 & 商 & - & & & & & & & & & & & & & & & & & & 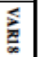 \\
\hline$\frac{\circ}{8}$ & 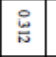 & 兽 & $\frac{\circ}{\bar{x}}$ & 活 & 䔬 & \begin{tabular}{|l|l|}
\multirow{3}{*}{} \\
\end{tabular} & $\frac{0}{9}$ & 员 & 离 & $\begin{array}{ll}0 \\
\vdots\end{array}$ & 总 & $\stackrel{9}{8}$ & 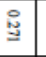 & $\stackrel{q}{q}$ & is & \&્छ & 兽 & 音 & $\frac{2}{a}$ & - & & & & & & & & & & & & & & & & & & & 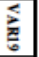 \\
\hline$\frac{\circ}{8}$ & \begin{tabular}{|l|}
$\circ$ \\
\end{tabular} & 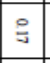 & 8 & 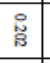 & \begin{tabular}{|l|}
$\bar{B}$ \\
\end{tabular} & \begin{tabular}{|l|l|}
$\frac{1}{3}$ \\
\end{tabular} & $\dot{8}$ & $\frac{\vdots}{1}$ & 舜 & 영 & 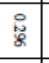 & \% & 覓 & 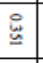 & 虫 & 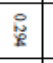 & 妾 & $\begin{array}{ll}+1 \\
3\end{array}$ & - & & & & & & & & & & & & & & & & & & & & 产 \\
\hline$\frac{2}{z}$ & : & 咅 & $\frac{8}{4}$ & 岁 & 芯 & $\frac{8}{\frac{8}{2}}$ & $\underline{\underline{\underline{Z}}}$ & $\frac{8}{0}$ & $\underline{\underline{E}}$ & & $\stackrel{\circ}{\Xi}$ & $\stackrel{Z}{Z}$ & 意 & 嚷 & $\frac{8}{ \pm}$ & $\stackrel{\mathscr{m}}{\mathrm{m}}$ & 主 & - & & & & & & & & & & & & & & & & & & & & & 彦 \\
\hline$\stackrel{B}{*}$ & 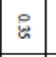 & $\stackrel{\circ}{\circ}$ & 弯 & 惫 & $\stackrel{1}{-1}$ & $\begin{array}{ll} \\
\dot{*}\end{array}$ & 票 & 岕 & 高 & 辛 & $\stackrel{\Xi}{\mathscr{E}}$ & $\stackrel{8}{\circ}$ & $\stackrel{\leftrightarrow}{s}$ & 变 & $\begin{array}{l}0 \\
b \\
\end{array}$ & 音 & - & & & & & & & & & & & & & & & & & & & & & & 预 \\
\hline is & 8 & $\begin{array}{l}8 \\
8 \\
8\end{array}$ & $\stackrel{G}{G}$ & $\stackrel{8}{8}$ & $\stackrel{\circ}{\circ}$ & : & $\stackrel{\circ}{\circ}$ & 总 & \& & 要 & : & $\frac{\mathscr{O}}{\Delta}$ & 密 & $\$$ & 焉 & - & & & & & & & & & & & & & & & & & & & & & & & 总 \\
\hline 要 & 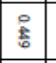 & \begin{tabular}{|l|l|}
$\xi$ \\
\end{tabular} & 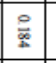 & 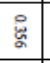 & : & \begin{tabular}{|l|l|}
\multirow{2}{*}{} \\
\end{tabular} & 总 & 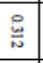 & 善 & : & 高 & $\begin{array}{ll}8 \\
2\end{array}$ & $\underline{\underline{\underline{g}}}$ & $\underline{\underline{\underline{\underline{q}}}}$ & - & & & & & & & & & & & & & & & & & & & & & & & & 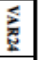 \\
\hline 8 & $\begin{array}{ll}\% \\
\end{array}$ & 荘 & $\stackrel{\circ}{\bar{L}}$ & ㅇ & $\frac{\circ}{9}$ & $\frac{O D}{4}$ & D & $\stackrel{8}{*}$ & 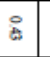 & 要 & $\&$ & $\stackrel{0}{0}$ & 善 & - & & & & & & & & & & & & & & & & & & & & & & & & & 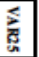 \\
\hline $\begin{array}{l} \\
\end{array}$ & : & \begin{tabular}{|l|}
3 \\
\end{tabular} & \begin{tabular}{|l|l|}
$\frac{1}{3}$ \\
\end{tabular} & 总 & \begin{tabular}{|l|l|}
8 \\
8
\end{tabular} & $\frac{2}{11}$ & 8 & 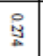 & 8 & i & 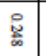 & 产 & - & & & & & & & & & & & & & & & & & & & & & & & & & & 结 \\
\hline : & \begin{tabular}{|l|l|}
8 \\
8
\end{tabular} & \begin{tabular}{|l|l|}
8 \\
8
\end{tabular} & \begin{tabular}{|l|l|}
\multirow{2}{*}{} \\
\end{tabular} & $\frac{1}{8}$ & \begin{tabular}{|l|l|}
8 \\
8
\end{tabular} & $\div$ & 通 & ¿ & 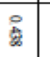 & 商 & 安 & - & & & & & & & & & & & & & & & & & & & & & & & & & & & 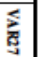 \\
\hline 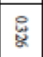 & \begin{tabular}{ll}
\multirow{8}{*}{} \\
\end{tabular} & $\begin{array}{ll}\stackrel{9}{=} \\
\end{array}$ & \begin{tabular}{|l|l|}
8 \\
8
\end{tabular} & 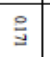 & \begin{tabular}{|l|}
$\frac{2}{4}$ \\
\end{tabular} & \% & : & 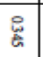 & 虽 & 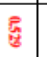 & . & & & & & & & & & & & & & & & & & & & & & & & & & & & & 产 \\
\hline$\frac{\circ}{1}$ & \begin{tabular}{|l|} 
\\
\\
\multirow{L}{*}{} \\
\end{tabular} & \begin{tabular}{|l|}
$\%$ \\
\end{tabular} & \begin{tabular}{|l|l|}
$\frac{O}{b}$ \\
\end{tabular} & $\frac{\circ}{\bar{c}}$ & \begin{tabular}{|l|}
\multirow{8}{*}{} \\
\end{tabular} & \begin{tabular}{|l|l|}
\multirow{2}{*}{} \\
\end{tabular} & $\begin{array}{ll} \\
\end{array}$ & $\%$ & 离 & - & & & & & & & & & & & & & & & & & & & & & & & & & & & & & 产 \\
\hline $\begin{array}{ll}\frac{2}{4} \\
\end{array}$ & \begin{tabular}{|l|l|}
\multirow{3}{*}{} \\
\end{tabular} & : & 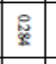 & \begin{tabular}{l|l}
$: 8$ \\
8
\end{tabular} & $\frac{\circ}{4}$ & \begin{tabular}{|l|l|}
\multirow{3}{*}{} \\
\end{tabular} & 落 & \begin{tabular}{|l|}
$\frac{\mathrm{g}}{\mathrm{w}}$ \\
\end{tabular} & - & & & & & & & & & & & & & & & & & & & & & & & & & & & & & & 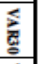 \\
\hline \begin{tabular}{|l}
$\mathscr{Q}$ \\
\end{tabular} & \begin{tabular}{|l|}
$\dddot{2}$ \\
\end{tabular} & \begin{tabular}{|l|} 
\\
\end{tabular} & \begin{tabular}{|l}
$\mathscr{8}$ \\
\end{tabular} & $\dot{q}$ & 㩊 & : & 爱 & - & & & & & & & & & & & & & & & & & & & & & & & & & & & & & & & $\underline{\underline{t}}$ \\
\hline$\frac{1}{4}$ & : & $\frac{1}{0}$ & 恋 & 过 & 8 & \begin{tabular}{|l|l|}
$y_{3}$ \\
\end{tabular} & - & & & & & & & & & & & & & & & & & & & & & & & & & & & & & & & & 鹿 \\
\hline 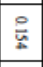 & \begin{tabular}{|l|l|}
$\circ$ \\
\end{tabular} & \begin{tabular}{|l|}
$\circ$ \\
\end{tabular} & \begin{tabular}{|l|} 
\\
\end{tabular} & 8 & $\therefore$ & - & & & & & & & & & & & & & & & & & & & & & & & & & & & & & & & & & 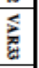 \\
\hline : & : & \begin{tabular}{|l|} 
\\
\end{tabular} & \& & : & - & & & & & & & & & & & & & & & & & & & & & & & & & & & & & & & & & & 委 \\
\hline$\underline{9}$ & 递 & : & $\frac{\Xi}{2}$ & - & & & & & & & & & & & & & & & & & & & & & & & & & & & & & & & & & & & 蛋 \\
\hline$\frac{1}{5}$ & 号 & $\vdots$ & - & & & & & & & & & & & & & & & & & & & & & & & & & & & & & & & & & & & & 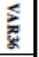 \\
\hline$\frac{2}{6}$ & 吉 & - & & & & & & & & & & & & & & & & & & & & & & & & & & & & & & & & & & & & & 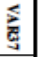 \\
\hline 离 & - & & & & & & & & & & & & & & & & & & & & & & & & & & & & & & & & & & & & & & 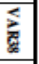 \\
\hline- & & & & & & & & & & & & & & & & & & & & & & & & & & & & & & & & & & & & & & & tz. \\
\hline
\end{tabular}




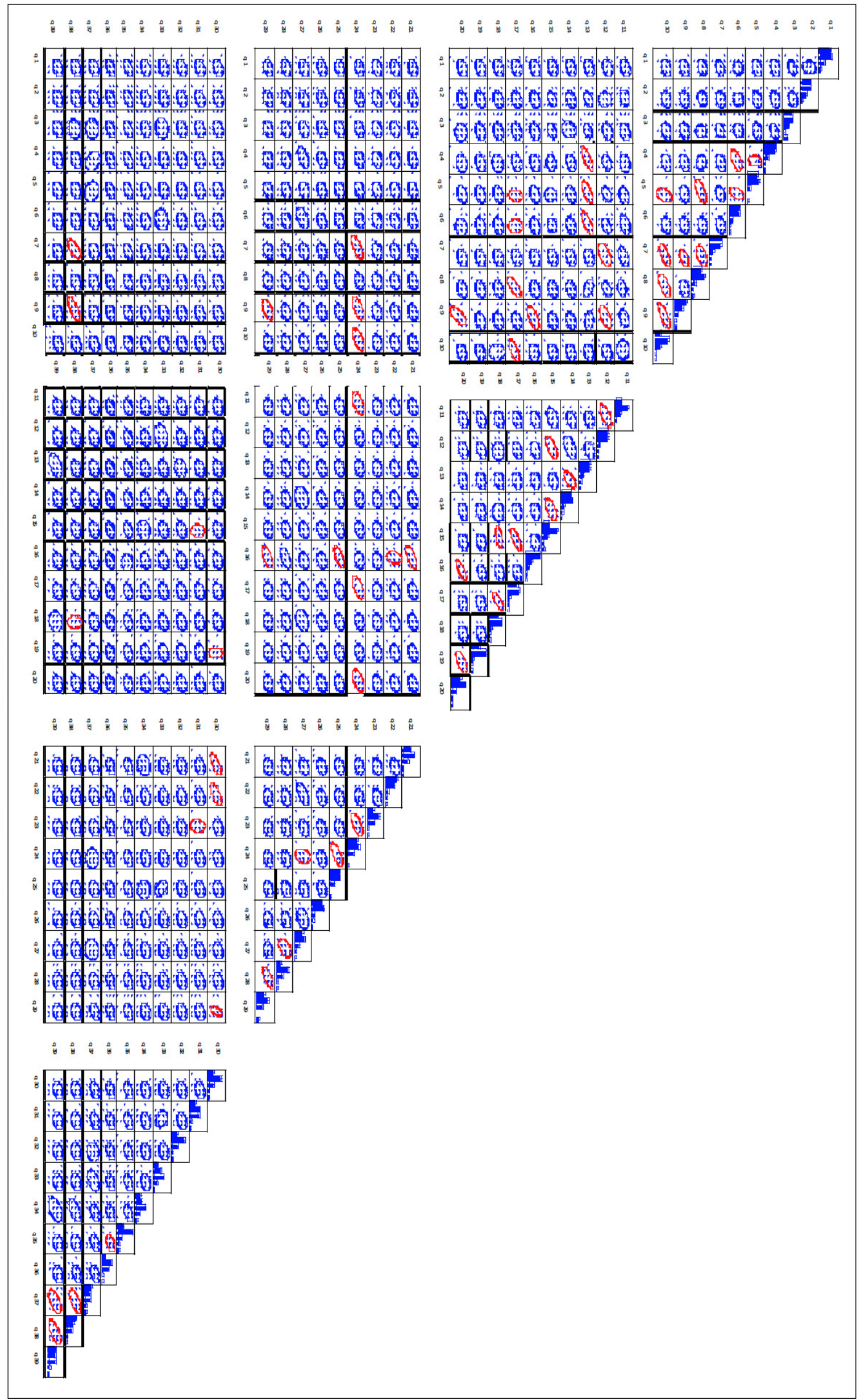

Fig. (6). Scatter plot matrix for the managerial questions. 
The correlation matrix among the variables was very large and is shown in Table 5b. The correlation matrix shows that most of the correlations were $<0.5$. About 50 of the correlations were between 0.5 and $<0.6$; six correlations were between 0.6 and $<0.7$, i.e., $\mathrm{q} 3$ and $\mathrm{q} 6, \mathrm{q} 8$ and $\mathrm{q} 17, \mathrm{q} 15$ and $\mathrm{q} 17, \mathrm{q} 17$ and $\mathrm{q} 18, \mathrm{q} 22$ and $\mathrm{q} 30$, and $\mathrm{q} 37$ and $\mathrm{q} 38$; the three highest correlations were between 0.7 and $<0.8$, i.e., q5 and $\mathrm{q} 6, \mathrm{q} 9$ and $\mathrm{q} 20$, and $\mathrm{q} 10$ and $\mathrm{q} 24$.

These correlations are also reflected in the scatter plot shown in (Fig. 6).

\section{CONCLUSION AND RECOMMENDATIONS}

The objective of this study was to identify the obstacles faced by the contractors sector in Saudi Arabia, where the data were analyzed using Spearman's [9] rank correlation method. The data represented the views of contractors as 71 responded to 74 questions by filling a simple digital interactive questionnaire to collect data from them. The study showed that the Likert-based sampling method described [11] was suitable for measuring the opinions of contractors and identified important factors facing them. It should be noted that these data were collected from the contractors themselves, without any input from clients or consultants. The results obtained using different sets of questions are presented as tables and figures, which were analyzed using Spearman's [9] rank correlation method.

It is recommended that further investigations should address the factors that underlie the obstacles faced by the contractors sector, including analyzing the views of designers/consultants and subcontractors/manufacturers.

\section{CONFLICT OF INTEREST}

The author(s) confirm that this article content has no conflicts of interest.

\section{ACKNOWLEDGEMENT}

This study was supported by the University of Dammam, Saudi Arabia Grant No. 2011104. The author acknowledges
Professor Sulaiman Bah for statistical analysis support and appreciates the contributions made by the team who worked on this project. Thanks are also extended to the contractors who participated by completing the questionnaires.

\section{REFERENCES}

[1] A. Al-Barrak, "Causes of Contractors' Failures in Saudi Arabia", Master of Science thesis, KFUPM, Dhahran, Saudi Arabia. June, 1993.

[2] N. Banaitienè, and A. Banaitis, "Analysis of criteria for contractors qualification evaluation", Technological and Economical Development of Economy, vol. XII, no. 4, pp. 276-282, 2006.

[3] J. Ahcom, "A Model for Benchmarking Contractors Project Management Elements in Saudi Arabia", Master of Science thesis, KFUPM, Dhahran, Saudi Arabia, May, 2004.

[4] K. Lam, T. Hu, and S. Ng, "Using the principal component analysis method as a tool in contractor pre-qualification", Construction Management and Economics, vol. 23, issue, September, pp. 673684, 2005.

[5] K. Al-Dughaither, "A multi-criteria decision making model for contractors pre-qualification", Joint International Conference on Computing and Decision Making in Civil Engineering, pp. 11221134, June 14-16, Montréal, Canada, 2006.

[6] W. Didibhuku, T. Thwawa, and M. Mvubu, "Problems facing small and medium size contractors in Swaziland", Journal Service and Management, vol. 2, pp. 253-361, 2009.

[7] N. Dmaidi, M. Dwaikat, and I. Shweikt, "Construction contracting management obstacles in Palestine", International Journal of Construction and Management, vol. 2, no. 1, pp. 15-22, 2013.

[8] I. Mahamid, "Causes of contractors' failure: contractors' view", $2^{\text {nd }}$ International Conference on Construction and Project Management (IPEDR), vol. 15, pp. 1-5. IACSIT Press, Singapore, 2011.

[9] R. B. Kariyam, and R. B. Perdana, "Factors of ordinal data weight ranking and its application to reduce perception variables to math lessons of senior high school student", In: Proceedings International Seminar and the Fourth National Conference on Mathematics Education "Building the Nation Character through Humanistic Mathematics Education", Department of Mathematics Education, Yogyakarta State University, Yogyakarta, July 21-21, 2011.

[10] Available at http://www.momra.gov.sa/, Ministry of Municipal and Rural Affairs, viewed July 2013.

[11] D. Bertram, Likert Scale, available at http://poincare.matf. bg.ac.rs/ kristina//topic-dane-likert.pdf, CPSC 681-Topic Report, pp. 1-10. 2006.

[12] Available at http://www.systat.com/MystatProducts.aspx, Systat Inc., viewed July 2013. 